\title{
The Dynamical Analysis of a Prey-Predator Model with a Refuge-Stage Structure Prey Population
}

\author{
Raid Kamel Naji ${ }^{1}$ and Salam Jasim Majeed ${ }^{1,2}$ \\ ${ }^{1}$ Department of Mathematics, College of Science, Baghdad University, Baghdad, Iraq \\ ${ }^{2}$ Department of Physics, College of Science, Thi-Qar University, Nasiriyah, Iraq \\ Correspondence should be addressed to Salam Jasim Majeed; sm.salammajeed@yahoo.com
}

Received 20 July 2016; Accepted 1 November 2016

Academic Editor: Jaume Giné

Copyright (c) 2016 R. K. Naji and S. J. Majeed. This is an open access article distributed under the Creative Commons Attribution License, which permits unrestricted use, distribution, and reproduction in any medium, provided the original work is properly cited.

\begin{abstract}
We proposed and analyzed a mathematical model dealing with two species of prey-predator system. It is assumed that the prey is a stage structure population consisting of two compartments known as immature prey and mature prey. It has a refuge capability as a defensive property against the predation. The existence, uniqueness, and boundedness of the solution of the proposed model are discussed. All the feasible equilibrium points are determined. The local and global stability analysis of them are investigated. The occurrence of local bifurcation (such as saddle node, transcritical, and pitchfork) near each of the equilibrium points is studied. Finally, numerical simulations are given to support the analytic results.
\end{abstract}

\section{Introduction}

The development of the qualitative analysis of ordinary differential equations is deriving to study many problems in mathematical biology. The modeling for the population dynamics of a prey-predator system is one of the important and interesting goals in mathematical biology, which has received wide attention by several authors [1-6]. In the natural world many kinds of prey and predator species have a life history that is composed of at least two stages: immature and mature, and each stage has different behavioral properties. So, some works of stage structure prey-predator models have been provided in a good number of papers in the literatures [7-12]. Zhang et al. in [9] and Cui and Takeuchi in [11] proposed two mathematical models of prey stage structure; in these models the predator species consumes exclusively the immature prey. Indeed, there are many factors that impact the dynamics of prey-predator interactions such as disease, harvesting, prey refuge, delay, and many other factors. Several prey species have gone to extinction, and this extinction must be caused by external effects such as overutilization, overpredation, and environmental factors (pollution, famine). Prey may avoid becoming attacked by predators either by protecting themselves or by living in a refuge where it will be out of sight of predators. Some theoretical and empirical studies have shown and tested the effects of prey refuges and making an opinion that the refuges used by prey have a stabilizing influence on preypredator interactions; also prey extinction can be prevented by the addition of refuges; for instance, we can refer to [13-23]. Therefore, it is important and worthwhile to study the effects of a refuge on the prey population with prey stage structure. Consequently, in this paper, we proposed and analyzed the prey-predator model involving a stage structure in prey population together with a preys refuge property as a defensive property against the predation.

\section{Mathematical Model}

In this section a prey-predator model with a refuge-stage structure prey population is proposed for study. Let $x(T)$ represent the population size of the immature prey at time $T ; y(T)$ represents the population size of the mature prey at time $T$, while $z(T)$ denotes the population size of the predator species at time $T$. Therefore in order to describe the dynamics 
of this model mathematically the following hypotheses are adopted:

(1) The immature prey grows exponentially depending completely on its parents with growth rate $r>0$. There is an intraspecific competition between their individuals with intraspecific competition rate $\delta_{1}>0$. The immature prey individual becomes mature with grownup rate $\beta>0$ and faces natural death with a rate $d_{1}>0$.

(2) There is an intraspecific competition between the individuals of mature prey population with intraspecific competition rate $\delta_{2}>0$. Further the mature prey species faces natural death rate too with a rate $d_{2}>0$.

(3) The environment provides partial protection of prey species against the predation with a refuge rate $0<$ $m<1$; therefore there is $1-m$ of prey species available for predation.

(4) There is an intraspecific competition between the individuals of predator population with intraspecific competition rate $\delta_{3}>0$. Further the predator species faces natural death rate too with a rate $d_{3}>0$.

The predator consumes the prey in both the compartments according to the mass action law represented by LotkaVolterra type of functional response with conversion rates $0<e_{1}<1$ and $0<e_{2}<1$ for immature and mature prey, respectively. Consequently, the dynamics of this model can be represented mathematically with the following set of differential equations.

$$
\begin{aligned}
& \dot{x}=r y-\delta_{1} x^{2}-d_{1} x-\beta x-\gamma_{1}(1-m) x z \\
& \dot{y}=\beta x-\delta_{2} y^{2}-d_{2} y-\gamma_{2}(1-m) y z \\
& \dot{z}=e_{1} \gamma_{1}(1-m) x z+e_{2} \gamma_{2}(1-m) y z-\delta_{3} z^{2}-d_{3} z
\end{aligned}
$$

with initial conditions, $x(0)>0, y(0)>0$, and $z(0)>0$.

In order to simplify the analysis of system (1) the number of parameters in system (1) is reduced using the following dimensionless variables in system (1):

$$
\begin{aligned}
y_{1} & =\frac{e_{1} \gamma_{1}(1-m)}{d_{2}} x, \\
y_{2} & =\frac{e_{2} \gamma_{2}(1-m)}{d_{2}} y, \\
y_{3} & =\frac{\delta_{3}}{d_{2}} z, \\
t & =d_{2} T .
\end{aligned}
$$

Accordingly, the dimensionless form of system (1) can be written as

$$
\begin{aligned}
& \dot{y}_{1}=a_{1} y_{2}-a_{2} y_{1}^{2}-a_{3} y_{1}-a_{4} y_{1} y_{3} \\
& \dot{y}_{2}=b_{1} y_{1}-b_{2} y_{2}^{2}-y_{2}-b_{3} y_{2} y_{3} \\
& \dot{y}_{3}=y_{1} y_{3}+y_{2} y_{3}-y_{3}^{2}-b_{4} y_{3},
\end{aligned}
$$

where the dimensionless parameters are given by

$$
\begin{aligned}
& a_{1}=\frac{e_{1} \gamma_{1} r}{e_{2} \gamma_{2} d_{2}}, \\
& a_{2}=\frac{\delta_{1}}{e_{1} \gamma_{1}(1-m)}, \\
& a_{3}=\frac{d_{1}+\beta}{d_{2}}, \\
& a_{4}=\frac{\gamma_{1}(1-m)}{\delta_{3}}, \\
& b_{1}=\frac{e_{2} \gamma_{2} \beta}{e_{1} \gamma_{1} d_{2}}, \\
& b_{2}=\frac{\delta_{2}}{e_{2} \gamma_{2}(1-m)}, \\
& b_{3}=\frac{\gamma_{2}(1-m)}{\delta_{3}}, \\
& b_{4}=\frac{d_{3}}{d_{2}} .
\end{aligned}
$$

According to the equations given in system (3), all the interaction functions are continuous and have a continuous partial derivatives. Therefore they are Lipschitzain and hence the solution of system (3) exists and is unique. Moreover the solution of system (3) is bounded as shown in the following theorem.

Theorem 1. All the solutions of system (3) that initiate in the positive octant are uniformly bounded.

Proof. Let $W=y_{1}+y_{2}+y_{3}$ be solutions of system (3) with initial conditions, $y_{1}(0)>0, y_{2}(0)>0$, and $y_{3}(0)>0$. Then by differentiation $W$ with respect to $t$ we get

$$
\begin{aligned}
\frac{d W}{d t} & \leq a_{1} y_{2}\left(1-\frac{y_{2}}{a_{1} / b_{2}}\right)+b_{1} y_{1}\left(1-\frac{y_{1}}{b_{1} / a_{2}}\right)-\sigma_{1} W \\
& \leq \sigma_{2}-\sigma_{1} W
\end{aligned}
$$

here $\sigma_{1}=\min \left\{1, a_{3}, b_{4}\right\}$ and $\sigma_{2}=a_{1}^{2} / 4 b_{2}+b_{1}^{2} / 4 a_{2}$. Now by using comparison theorem, we get

$$
0<W \leq\left(W_{0} e^{-\sigma_{1} t}+\frac{\sigma_{2}}{\sigma_{1}}\left(1-e^{-\sigma_{1} t}\right)\right) .
$$

Thus for $t \rightarrow \infty$ we obtain $0<W \leq \sigma_{2} / \sigma_{1}$. Hence, all solutions of system (3) in $R_{+}^{3}$ are uniformly bounded and therefore we have finished the proof.

\section{Local Stability Analysis}

It is observed that system (3) has at most three biologically feasible equilibrium points; namely, $E_{i}, i=0,1,2$. The existence conditions for each of these equilibrium points are discussed below: 
(1) The trivial equilibrium points $E_{0}=(0,0,0)$ exist always.

(2) The predator free equilibrium point is denoted by $E_{1}=\left(\tilde{y}_{1}, \tilde{y}_{2}, 0\right)$, where

$$
\tilde{y}_{1}=\frac{1}{b_{1}}\left(b_{2} \tilde{y}_{2}^{2}+\tilde{y}_{2}\right)
$$

while $\widetilde{y}_{2}$ is a positive root of the following third-order polynomial

$$
A_{1} y_{2}^{3}+A_{2} y_{2}^{2}+A_{3} y_{2}+A_{4}=0
$$

here, $A_{1}=a_{2} b_{2}^{2} / b_{1}^{2}, A_{2}=2 a_{2} b_{2} / b_{1}^{2}, A_{3}=\left(a_{2}+\right.$ $\left.a_{3} b_{1} b_{2}\right) / b_{1}^{2}$, and $A_{4}=\left(a_{3}-a_{1} b_{1}\right) / b_{1}$ exist uniquely in the interior of $y_{1} y_{2}$-plane if and only if the following condition holds:

$$
a_{3}<a_{1} b_{1} \text {. }
$$

(3) The interior (positive) equilibrium point is given by $E_{2}=\left(y_{1}^{*}, y_{2}^{*}, y_{3}^{*}\right)$, where

$$
\begin{aligned}
& y_{1}^{*}=\frac{\left[\left(b_{2}+b_{3}\right) y_{2}^{*}+\left(1-b_{3} b_{4}\right)\right] y_{2}^{*}}{b_{1}-b_{3} y_{2}^{*}}, \\
& y_{3}^{*}=y_{1}^{*}+y_{2}^{*}-b_{4}
\end{aligned}
$$

while $y_{2}^{*}$ is a positive root of the following third-order polynomial:

$$
B_{1} y_{2}^{3}+B_{2} y_{2}^{2}+B_{3} y_{2}+B_{4}=0
$$

here,

$$
\begin{aligned}
B_{1}= & a_{2}\left(b_{2}+b_{3}\right)^{2}+a_{4} b_{2}\left(b_{2}+b_{3}\right)>0, \\
B_{2}= & {\left[2 a_{2}\left(b_{2}+b_{3}\right)+2 a_{4} b_{2}+a_{4} b_{3}\right]\left(1-b_{3} b_{4}\right) } \\
& -\left[a_{3} b_{2} b_{3}+b_{3}^{2}\left(a_{3}+a_{1}\right)\right], \\
B_{3}= & 2 a_{1} b_{1} b_{3}+\left(a_{2}+a_{4}\right)\left(1-b_{3} b_{4}\right)^{2} \\
& +b_{1}\left(a_{3}-a_{4} b_{4}\right)\left(b_{2}+b_{3}\right) \\
& +\left[b_{1} a_{4}-b_{3}\left(a_{3}-a_{4} b_{4}\right)\right]\left(1-b_{3} b_{4}\right), \\
B_{4}= & b_{1}\left[a_{3}-a_{1} b_{1}-a_{3} b_{3} b_{4}-a_{4} b_{4}\left(1-b_{3} b_{4}\right)\right] .
\end{aligned}
$$

Clearly, (11) has a unique positive root represented by $y_{2}^{*}$ if the following set of conditions hold:

$$
B_{4}<0 \text { with }\left(B_{2}>0 \text { or } B_{3}<0\right)
$$

Therefore, $E_{2}$ exists uniquely in int. $R_{+}^{3}$ if in addition to condition (13) the following conditions are satisfied.

$$
\begin{aligned}
y_{1}^{*}+y_{2}^{*}>b_{4} \\
\frac{b_{3} b_{4}-1}{b_{2}+b_{3}}<y_{2}^{*}<\frac{b_{1}}{b_{3}} \\
\text { or } \frac{b_{1}}{b_{3}}<y_{2}^{*}<\frac{b_{3} b_{4}-1}{b_{2}+b_{3}} .
\end{aligned}
$$

Now to study the local stability of these equilibrium points, the Jacobian matrix $J\left(y_{1}, y_{2}, y_{3}\right)$ for the system (3) at any point $\left(y_{1}, y_{2}, y_{3}\right)$ is determined as

$$
\left(\begin{array}{ccc}
-2 a_{2} y_{1}-a_{3}-a_{4} y_{3} & a_{1} & -a_{4} y_{1} \\
b_{1} & -2 b_{2} y_{2}-1-b_{3} y_{3} & -b_{3} y_{2} \\
y_{3} & y_{3} & y_{1}+y_{2}-2 y_{3}-b_{4}
\end{array}\right) .
$$

Thus, system (3) has the following Jacobian matrix near $E_{0}=$ $(0,0,0)$.

$$
J\left(E_{0}\right)=\left(\begin{array}{ccc}
-a_{3} & a_{1} & 0 \\
b_{1} & -1 & 0 \\
0 & 0 & -b_{4}
\end{array}\right)
$$

Then the characteristic equation of $J\left(E_{0}\right)$ is given by

$$
\left(\lambda+b_{4}\right)\left[\lambda^{2}+\left(a_{3}+1\right) \lambda+a_{3}-a_{1} b_{1}\right]=0 .
$$

Clearly, all roots of (17) have negative real parts if and only if the following condition holds:

$$
a_{3}>a_{1} b_{1}
$$

So, $E_{0}$ is locally asymptotically stable under condition (18) and saddle point otherwise. Therefore, $E_{0}$ is locally asymptotically stable whenever $E_{1}$ does not exist and unstable whenever $E_{1}$ exists.

The Jacobian matrix of system (3) around the equilibrium point $E_{1}=\left(\tilde{y}_{1}, \widetilde{y}_{2}, 0\right)$ reduced to

$$
\begin{aligned}
J\left(E_{1}\right) & =\left(\begin{array}{ccc}
-2 a_{2} \tilde{y}_{1}-a_{3} & a_{1} & -a_{4} \tilde{y}_{1} \\
b_{1} & -2 b_{2} \tilde{y}_{2}-1 & -b_{3} \tilde{y}_{2} \\
0 & 0 & \tilde{y}_{1}+\tilde{y}_{2}-b_{4}
\end{array}\right) \\
& =\left(a_{i j}\right)_{3 \times 3} .
\end{aligned}
$$

Then the characteristic equation of $J\left(E_{1}\right)$ is written by

$$
\left[\lambda-a_{33}\right]\left[\lambda^{2}-\left(a_{11}+a_{22}\right) \lambda+a_{11} a_{22}-a_{12} a_{21}\right]=0 .
$$

Straightforward computation shows that all roots of (20) have negative real part provided that

$$
\begin{aligned}
\tilde{y}_{1}+\tilde{y}_{2} & <b_{4} \\
\left(2 a_{2} \tilde{y}_{1}+a_{3}\right)\left(2 b_{2} \tilde{y}_{2}+1\right) & >a_{1} b_{1} .
\end{aligned}
$$

So, $E_{1}$ is locally asymptotically stable if the above two conditions hold.

Finally the Jacobian matrix of system (3) around the interior equilibrium point $E_{2}=\left(y_{1}^{*}, y_{2}^{*}, y_{3}^{*}\right)$ is written

$$
J\left(E_{2}\right)=\left(b_{i j}\right)_{3 \times 3}
$$


here

$$
\begin{aligned}
& b_{11}=-\left(2 a_{2} y_{1}^{*}+a_{4} y_{3}^{*}+a_{3}\right), \\
& b_{12}=a_{1} \\
& b_{13}=-a_{4} y_{1}^{*} \\
& b_{21}=b_{1} \\
& b_{22}=-\left(2 b_{2} y_{2}^{*}+b_{3} y_{3}^{*}+1\right), \\
& b_{23}=-b_{3} y_{2}^{*} \\
& b_{31}=y_{3}^{*} \\
& b_{32}=y_{3}^{*} \\
& b_{33}=-y_{3}^{*} .
\end{aligned}
$$

Hence, the characteristic equation of $J\left(E_{2}\right)$ becomes

$$
\lambda^{3}+D_{1} \lambda^{2}+D_{2} \lambda+D_{3}=0
$$

with

$$
\begin{aligned}
D_{1}= & -\left(b_{11}+b_{22}+b_{33}\right)>0, \\
D_{2}= & b_{11} b_{22}-b_{12} b_{21}+b_{11} b_{33}-b_{13} b_{31}+b_{22} b_{33} \\
& -b_{23} b_{32}, \\
D_{3}= & b_{33}\left(b_{12} b_{21}-b_{11} b_{22}\right)+b_{11} b_{23} b_{32}-b_{12} b_{23} b_{31} \\
& +b_{22} b_{13} b_{31}-b_{21} b_{13} b_{32} .
\end{aligned}
$$

Consequently, $\Delta=D_{1} D_{2}-D_{3}$ can be written as

$$
\begin{aligned}
\Delta & =\left(b_{11}+b_{22}\right)\left(b_{12} b_{21}-b_{11} b_{22}\right)-b_{11} b_{22} b_{33} \\
& -\left(b_{11}+b_{22}\right)\left(b_{11} b_{33}-b_{13} b_{31}\right) . \\
- & b_{22}^{2} b_{33}-b_{11} b_{33}^{2}-b_{22} b_{33}^{2}+b_{13} b_{31}\left(b_{33}+b_{21}\right) \\
& +b_{23} b_{32}\left(b_{33}+b_{12}\right)
\end{aligned}
$$

Since $D_{1}>0$, then according to Routh-Hurwitz criterion $E_{2}$ is locally asymptotically stable if and only if $D_{3}>0$ and $\Delta=$ $D_{1} D_{2}-D_{3}>0$. According to the form of $D_{3}$ and the signs of Jacobian elements the last four terms are positive, while the first term will be positive under the sufficient condition (28) below. However $\Delta$ becomes positive if and only if in addition to condition (28) the second sufficient condition given by (29) holds.

$$
\begin{aligned}
\left(2 a_{2} y_{1}^{*}+a_{4} y_{3}^{*}+a_{3}\right)\left(2 b_{2} y_{2}^{*}+b_{3} y_{3}^{*}+1\right) & >a_{1} b_{1} \\
y_{3}^{*} & >b_{1}, \\
y_{3}^{*} & >a_{1} .
\end{aligned}
$$

Therefore under these two sufficient conditions $E_{2}$ is locally asymptotically stable.

\section{Global Stability}

In this section the global stability for the equilibrium points of system (3) is investigated by using the Lyapunov method as shown in the following theorems.

Theorem 2. Assume that the vanishing equilibrium point $E_{0}$ is locally asymptotically stable; then it is globally asymptotically stable in $R_{+}^{3}$ if and only if the following condition holds:

$$
\frac{a_{4} b_{1}}{a_{3}}<b_{3}<\frac{a_{4}}{a_{1}} \text {. }
$$

Proof. Consider the following positive definite real valued function:

$$
V_{0}\left(y_{1}, y_{2}, y_{3}\right)=\frac{1}{a_{4}} y_{1}+\frac{1}{b_{3}} y_{2}+y_{3}
$$

Straightforward computation shows that the derivative of $V_{0}$ with respect to $t$ is given by

$$
\frac{d V_{0}}{d t}<\left(\frac{a_{4} b_{1}-a_{3} b_{3}}{a_{4} b_{3}}\right) y_{1}+\left(\frac{a_{1} b_{3}-a_{4}}{a_{4} b_{3}}\right) y_{2}-b_{4} y_{3} .
$$

Therefore, by using condition (30), we obtain $d V_{0} / d t$ which is negative definite in $R_{+}^{3}$, and then $V_{0}$ is a Lyapunov function with respect to $E_{0}$. Hence $E_{0}$ is globally asymptotically stable in $R_{+}^{3}$ and the proof is complete.

Theorem 3. Assume that the predator free equilibrium point $E_{1}=\left(\tilde{y}_{1}, \tilde{y}_{2}, 0\right)$ is locally asymptotically stable; then it is globally asymptotically stable in $R_{+}^{3}$ if the following condition holds:

$$
\begin{aligned}
& \left(\frac{a_{1}}{a_{4} y_{1}}+\frac{b_{1}}{b_{3} y_{2}}\right)^{2} \\
& \quad<4\left(\frac{a_{1} \tilde{y}_{2}}{a_{4} y_{1} \tilde{y}_{1}}+\frac{a_{2}}{a_{4}}\right)\left(\frac{b_{1} \tilde{y}_{1}}{b_{3} y_{2} \tilde{y}_{2}}+\frac{b_{2}}{b_{3}}\right) .
\end{aligned}
$$

Proof. Consider the following positive definite real valued function:

$$
\begin{aligned}
V_{1}\left(y_{1}, y_{2}, y_{3}\right)= & \frac{1}{a_{4}}\left(y_{1}-\tilde{y}_{1}-\tilde{y}_{1} \ln \frac{y_{1}}{\tilde{y}_{1}}\right) \\
& +\frac{1}{b_{3}}\left(y_{2}-\tilde{y}_{2}-\tilde{y}_{2} \ln \frac{y_{2}}{\tilde{y}_{2}}\right)+y_{3} .
\end{aligned}
$$

Straightforward computation shows that the derivative of $V_{1}$ with respect to $t$ is given by

$$
\begin{aligned}
\frac{d V_{1}}{d t}= & -\left(\frac{a_{1} \tilde{y}_{2}}{a_{4} y_{1} \tilde{y}_{1}}+\frac{a_{2}}{a_{4}}\right)\left(y_{1}-\tilde{y}_{1}\right)^{2} \\
& -\left(\frac{b_{1} \tilde{y}_{1}}{b_{3} y_{2} \tilde{y}_{2}}+\frac{b_{2}}{b_{3}}\right)\left(y_{2}-\tilde{y}_{2}\right)^{2} \\
& +\left(\frac{a_{1}}{a_{4} y_{1}}+\frac{b_{1}}{b_{3} y_{2}}\right)\left(y_{1}-\tilde{y}_{1}\right)\left(y_{2}-\tilde{y}_{2}\right)-y_{3}^{2} \\
& -\left(b_{4}-\tilde{y}_{1}-\tilde{y}_{2}\right) y_{3} .
\end{aligned}
$$


Now using condition (33) gives us that

$$
\begin{aligned}
& \frac{d V_{1}}{d t}<-\left[\sqrt{\frac{a_{1} \tilde{y}_{2}}{a_{4} y_{1} \tilde{y}_{1}}+\frac{a_{2}}{a_{4}}}\left(y_{1}-\tilde{y}_{1}\right)\right. \\
& \left.\quad-\sqrt{\frac{b_{1} \tilde{y}_{1}}{b_{3} y_{2} \tilde{y}_{2}}+\frac{b_{2}}{b_{3}}}\left(y_{2}-\tilde{y}_{2}\right)\right]^{2}-\left(b_{4}-\tilde{y}_{1}-\tilde{y}_{2}\right) y_{3} .
\end{aligned}
$$

Clearly $d V_{1} / d t$ is negative definite due to local stability condition (21). Hence $V_{1}$ is a Lyapunov function with respect to $E_{1}$, and then $E_{1}$ is globally asymptotically stable, which completes the proof.

Theorem 4. Assume that the interior equilibrium point $E_{2}=$ $\left(y_{1}^{*}, y_{2}^{*}, y_{3}^{*}\right)$ is locally asymptotically stable in $R_{+}^{3}$; then it is globally asymptotically stable if and only if the following condition holds:

$$
\left(\frac{y_{2}^{*} a_{1} b_{3}}{y_{1}^{*} b_{1}}-a_{4}\right)^{2}<4 \frac{y_{2}^{*} a_{1} a_{2} b_{3} b_{4}}{y_{1}^{*} b_{1}} .
$$

Proof. Consider the following positive definite real valued function around $E_{2}$ :

$$
\begin{aligned}
V_{1}\left(y_{1}, y_{2}, y_{3}\right)= & \left(y_{1}-y_{1}^{*}-y_{1}^{*} \ln \frac{y_{1}}{y_{1}^{*}}\right) \\
& +\frac{y_{2}^{*} a_{1}}{y_{1}^{*} b_{1}}\left(y_{2}-y_{2}^{*}-y_{2}^{*} \ln \frac{y_{2}}{y_{2}^{*}}\right) \\
& +\frac{y_{2}^{*} a_{1} b_{3}}{y_{1}^{*} b_{1}}\left(y_{3}-y_{3}^{*}-y_{3}^{*} \ln \frac{y_{3}}{y_{3}^{*}}\right) .
\end{aligned}
$$

Our computation for the derivative of $V_{2}$ with respect to $t$ gives that

$$
\begin{aligned}
\frac{d V_{2}}{d t}= & -\frac{a_{1}}{y_{1} y_{2} y_{1}^{*}}\left(y_{2} y_{1}^{*}-y_{1} y_{2}^{*}\right)^{2}-a_{2}\left(y_{1}-y_{1}^{*}\right)^{2} \\
& -\frac{y_{2}^{*} a_{1} b_{2}}{y_{1}^{*} b_{1}}\left(y_{2}-y_{2}^{*}\right)^{2} \\
& +\left(\frac{y_{2}^{*} a_{1} b_{3}}{y_{1}^{*} b_{1}}-a_{4}\right)\left(y_{1}-y_{1}^{*}\right)\left(y_{3}-y_{3}^{*}\right) \\
& -\frac{y_{2}^{*} a_{1} b_{3} b_{4}}{y_{1}^{*} b_{1}}\left(y_{3}-y_{3}^{*}\right)^{2} .
\end{aligned}
$$

Now by using the condition (37) we obtain that

$$
\begin{aligned}
& \frac{d V_{2}}{d t} \\
& <-\frac{a_{1}}{y_{1} y_{2} y_{1}^{*}}\left(y_{2} y_{1}^{*}-y_{1} y_{2}^{*}\right)^{2}-\frac{y_{2}^{*} a_{1} b_{2}}{y_{1}^{*} b_{1}}\left(y_{2}-y_{2}^{*}\right)^{2} \\
& \quad-\left[\sqrt{a_{2}}\left(y_{1}-y_{1}^{*}\right)-\sqrt{\frac{y_{2}^{*} a_{1} b_{3} b_{4}}{y_{1}^{*} b_{1}}}\left(y_{3}-y_{3}^{*}\right)\right]^{2} .
\end{aligned}
$$

According to the above inequality we have $d V_{2} / d t$ which is negative definite; therefore, $E_{2}$ is globally asymptotically stable in $R_{+}^{3}$ and hence the proof is complete.

\section{Local Bifurcation}

In this section the local bifurcation near the equilibrium points of system (3) is investigated using Sotomayor's theorem for local bifurcation [24]. It is well known that the existence of nonhyperbolic equilibrium point is a necessary but not sufficient condition for bifurcation to occur. Now rewrite system (3) in the form

$$
\dot{Y}=f(Y)
$$

where

$$
\begin{gathered}
Y=\left(y_{1}, y_{2}, y_{3}\right)^{T}, \\
f=\left(f_{1}, f_{2}, f_{3}\right)^{T} .
\end{gathered}
$$

Then according to Jacobian matrix of system (3) given in (15), it is simple to verify that for any nonzero vector $U=$ $\left(u_{1}, u_{2}, u_{3}\right)^{T}$ we have

$$
\begin{aligned}
D^{2} f & \left(y_{1}, y_{2}, y_{3}\right)(U, U) \\
= & \frac{\partial^{2} f}{\partial y_{1}^{2}} u_{1}^{2}+\frac{\partial^{2} f}{\partial y_{1} \partial y_{2}} u_{1} u_{2}+\frac{\partial^{2} f}{\partial y_{2} \partial y_{1}} u_{2} u_{1}+\frac{\partial^{2} f}{\partial y_{2}^{2}} u_{2}^{2} \\
& +\frac{\partial^{2} f}{\partial y_{1} \partial y_{3}} u_{1} u_{3}+\frac{\partial^{2} f}{\partial y_{3} \partial y_{1}} u_{3} u_{1}+\frac{\partial^{2} f}{\partial y_{2} \partial y_{3}} u_{2} u_{3} \\
& +\frac{\partial^{2} f}{\partial y_{3} \partial y_{2}} u_{3} u_{2}+\frac{\partial^{2} f}{\partial y_{3}^{2}} u_{3}^{2} .
\end{aligned}
$$

Consequently, we obtain that

$$
D^{2} f\left(y_{1}, y_{2}, y_{3}\right)(U, U)=\left(\begin{array}{c}
-2 a_{2} u_{1}^{2}-2 a_{4} u_{1} u_{3} \\
-2 b_{2} u_{2}^{2}-2 b_{3} u_{2} u_{3} \\
2 u_{1} u_{3}+2 u_{2} u_{3}-2 u_{3}^{2}
\end{array}\right)
$$

and therefore

$$
D^{3} f\left(y_{1}, y_{2}, y_{3}\right)(U, U, U)=\left(\begin{array}{l}
0 \\
0 \\
0
\end{array}\right) .
$$

Thus system (3) has no pitchfork bifurcation due to (45). Moreover, the local bifurcation near the equilibrium points is investigated in the following theorems:

Theorem 5. System (3) undergoes a transcritical bifurcation near the vanishing equilibrium point, but saddle node bifurcation cannot occur, when the parameter $a_{3}$ passes through the bifurcation value $a_{3}^{*}=a_{1} b_{1}$.

Proof. According to the Jacobian matrix $J\left(E_{0}\right)$ given by (16), system (3) at the equilibrium point $E_{0}$ with $a_{3}=a_{3}^{*}$ has zero eigenvalue, say $\lambda_{0}^{*}=0$, and the Jacobian matrix $J\left(E_{0}, a_{3}^{*}\right)$ becomes

$$
J\left(E_{0}, a_{3}^{*}\right)=J_{0}^{*}=\left(\begin{array}{ccc}
-a_{3}^{*} & a_{1} & 0 \\
b_{1} & -1 & 0 \\
0 & 0 & -b_{4}
\end{array}\right)
$$


Now let $U^{[0]}=\left(u_{1}^{[0]}, u_{2}^{[0]}, u_{3}^{[0]}\right)^{T}$ be the eigenvector corresponding to the eigenvalue $\lambda_{0}^{*}=0$. Thus $J_{0}^{*} U^{[0]}=\mathbf{0}$ gives $U^{[0]}=\left(u_{1}^{[0]}, b_{1} u_{1}^{[0]}, 0\right)^{T}$, where $u_{1}^{[0]}$ represents any nonzero real number. Also, let $W^{[0]}=\left(w_{1}^{[0]}, w_{2}^{[0]}, w_{3}^{[0]}\right)^{T}$ represents the eigenvector corresponding to the eigenvalue $\lambda_{0}^{*}=0$ of $J_{0}^{* T}$. Hence $J_{0}^{* T} W^{[0]}=\mathbf{0}$ gives that $W^{[0]}=\left(w_{1}^{[0]}, a_{1} w_{1}^{[0]}, 0\right)^{T}$, where $w_{1}^{[0]}$ denotes any nonzero real number. Now, since

$$
\begin{aligned}
\frac{d f}{d a_{3}} & =f_{a_{3}}\left(Y, a_{3}\right)=\left(\frac{d f_{1}}{d a_{3}}, \frac{d f_{2}}{d a_{3}}, \frac{d f_{3}}{d a_{3}}\right)^{T} \\
& =\left(-y_{1}, 0,0\right)^{T}
\end{aligned}
$$

thus $f_{a_{3}}\left(E_{0}, a_{3}^{*}\right)=(0,0,0)^{T}$, which gives $\left(W^{[0]}\right)^{T} f_{a_{3}}\left(E_{0}, a_{3}^{*}\right)=$ 0 . So, according to Sotomayor's theorem for local bifurcation, system (3) has no saddle node bifurcation at $a_{3}=a_{3}^{*}$. Also, since

$$
D f_{a_{3}}\left(E_{0}, a_{3}\right)=\left(\begin{array}{ccc}
-1 & 0 & 0 \\
0 & 0 & 0 \\
0 & 0 & 0
\end{array}\right)
$$

then,

$$
\begin{aligned}
& \left(W^{[0]}\right)^{T}\left(D f_{a_{3}}\left(E_{0}, a_{3}^{*}\right) U^{[0]}\right) \\
& \quad=\left(w_{1}^{[0]}, a_{1} w_{1}^{[0]}, 0\right)\left(-u_{1}^{[0]}, 0,0\right)^{T}=-u_{1}^{[0]} w_{1}^{[0]} \neq 0 .
\end{aligned}
$$

Moreover, by substituting $E_{0}, a_{3}^{*}$, and $U^{[0]}$ in (44) we get that

$$
\begin{array}{rl}
D^{2} & f\left(E_{0}, a_{3}^{*}\right)\left(U^{[0]}, U^{[0]}\right) \\
& =\left(-2 a_{2}\left(u_{1}^{[0]}\right)^{2},-2 b_{2} b_{1}^{2}\left(u_{1}^{[0]}\right)^{2}, 0\right)^{T} .
\end{array}
$$

Hence, it is obtain that

$$
\begin{aligned}
& \left(W^{[0]}\right)^{T} D^{2} f\left(E_{0}, a_{3}^{*}\right)\left(U^{[0]}, U^{[0]}\right) \\
& =-2\left(a_{2}+a_{1} b_{2} b_{1}^{2}\right)\left(u_{1}^{[0]}\right)^{2} w_{1}^{[0]} \neq 0 .
\end{aligned}
$$

Thus, according to Sotomayor's theorem system (3) has a transcritical bifurcation at $E_{0}$ as the parameter $a_{3}$ passes through the value $a_{3}^{*}$; thus the proof is complete.

Theorem 6. Assume that condition (22) holds; then system (3) undergoes a transcritical bifurcation near the predator free equilibrium point $E_{1}$, but saddle node bifurcation cannot occur, when the parameter $b_{4}$ passes through the bifurcation value $b_{4}^{*}=\tilde{y}_{1}+\tilde{y}_{2}$.

Proof. According to the Jacobian matrix $J\left(E_{1}\right)$ given by (19), system (3) at the equilibrium point $E_{1}$ with $b_{4}=b_{4}^{*}$ has zero eigenvalue, say $\lambda_{1}^{*}=0$, and the Jacobian matrix $J\left(E_{1}, b_{4}^{*}\right)$ becomes

$$
J\left(E_{1}, b_{4}^{*}\right)=J_{1}^{*}=\left(a_{i j}^{*}\right)_{3 \times 3},
$$

where $a_{i j}^{*}=a_{i j} ; \forall i, j=1,2,3$, with $a_{33}^{*}=0$. Now let, $U^{[1]}=\left(u_{1}^{[1]}, u_{2}^{[1]}, u_{3}^{[1]}\right)^{T}$ be the eigenvector corresponding to the eigenvalue $\lambda_{1}^{*}=0$. Thus $J_{1}^{*} U^{[1]}=\mathbf{0}$ gives $U^{[1]}=$ $\left(\Lambda_{1} u_{3}^{[1]}, \Lambda_{2} u_{3}^{[1]}, u_{3}^{[1]}\right)^{T}$, where $\Lambda_{1}=\left(a_{12} a_{23}-a_{22} a_{13}\right) /\left(a_{11} a_{22}-\right.$ $\left.a_{12} a_{21}\right)$ and $\Lambda_{2}=\left(a_{21} a_{13}-a_{11} a_{31}\right) /\left(a_{11} a_{22}-a_{12} a_{21}\right)$ are negative according to the sign of the Jacobian elements and $u_{3}^{[1]}$ represents any nonzero real numbers. Also, let $W^{[1]}=$ $\left(w_{1}^{[1]}, w_{2}^{[1]}, w_{3}^{[1]}\right)^{T}$ represent the eigenvector corresponding to eigenvalue $\lambda_{1}^{*}=0$ of $J_{1}^{* T}$. Hence $J_{1}^{* T} W^{[1]}=\mathbf{0}$ gives that $W^{[1]}=\left(0,0, w_{3}^{[1]}\right)^{T}$, where $w_{3}^{[1]}$ stands for any nonzero real numbers. Now, since

$$
\frac{d f}{d b_{4}}=f_{b_{4}}\left(Y, b_{4}\right)=\left(\frac{d f_{1}}{d b_{4}}, \frac{d f_{2}}{d b_{4}}, \frac{d f_{3}}{d b_{4}}\right)^{T}=\left(0,0,-y_{3}\right)^{T}
$$

thus $f_{b_{4}}\left(E_{1}, b_{4}^{*}\right)=(0,0,0)^{T}$, which gives $\left(W^{[1]}\right)^{T} f_{b_{4}}\left(E_{1}, b_{4}^{*}\right)=$ 0 . So, according to Sotomayor's theorem for local bifurcation, system (3) has no saddle node bifurcation at $b_{4}=b_{4}^{*}$. Also, since

$$
D f_{b_{4}}\left(E_{1}, b_{4}\right)=\left(\begin{array}{ccc}
0 & 0 & 0 \\
0 & 0 & 0 \\
0 & 0 & -1
\end{array}\right)
$$

then, we can have

$$
\begin{aligned}
& \left(W^{[1]}\right)^{T}\left(D f_{b_{4}}\left(E_{1}, b_{4}^{*}\right) U^{[1]}\right) \\
& \quad=\left(0,0, w_{3}^{[1]}\right)\left(0,0,-u_{3}^{[1]}\right)^{T}=-u_{3}^{[1]} w_{3}^{[1]} \neq 0 .
\end{aligned}
$$

Moreover, substituting $E_{1}, b_{4}^{*}$, and $U^{[1]}$ in (44) gives

$$
\begin{aligned}
& D^{2} f\left(E_{1}, b_{4}^{*}\right)\left(U^{[1]}, U^{[1]}\right)=2\left(u_{3}^{[1]}\right)^{2} \\
& \quad \cdot\left(-a_{2} \Lambda_{1}^{2}-a_{4} \Lambda_{1},-b_{2} \Lambda_{2}^{2}-b_{3} \Lambda_{2}, \Lambda_{1}+\Lambda_{2}-1\right)^{T} .
\end{aligned}
$$

Hence, it is obtained that

$$
\begin{aligned}
& \left(W^{[1]}\right)^{T} D^{2} f\left(E_{1}, b_{4}^{*}\right)\left(U^{[1]}, U^{[1]}\right) \\
& \quad=2\left(\Lambda_{1}+\Lambda_{2}-1\right)\left(u_{3}^{[1]}\right)^{2} w_{3}^{[1]} \neq 0 .
\end{aligned}
$$

Thus, according to Sotomayor's theorem system (3) has a transcritical bifurcation at $E_{1}$ as the parameter $b_{4}$ passes through the value $b_{4}^{*}$; thus the proof is complete.

Theorem 7. Assume that condition (21) holds; then system (3) undergoes a saddle node bifurcation near the predator free equilibrium point $E_{1}$ when the parameter $a_{1}$ passes through the bifurcation value $a_{1}^{*}=\left(2 a_{2} \tilde{y}_{1}+a_{3}\right)\left(2 b_{2} \tilde{y}_{2}+1\right) / b_{1}$.

Proof. According to the Jacobian matrix $J\left(E_{1}\right)$ given by (19), system (3) at the equilibrium point $E_{1}$ with $a_{1}=a_{1}^{*}$ has zero eigenvalue, say $\lambda_{1}^{* *}=0$, and the Jacobian matrix $J\left(E_{1}, a_{1}^{*}\right)$ becomes

$$
J\left(E_{1}, a_{1}^{*}\right)=J_{1}^{* *}=\left(a_{i j}^{* *}\right)_{3 \times 3},
$$


where $a_{i j}^{* *}=a_{i j} ; \forall i, j=1,2,3$, with $a_{12}^{* *}=a_{1}^{*}$. Now let $U^{[11]}=\left(u_{1}^{[11]}, u_{2}^{[11]}, u_{3}^{[11]}\right)^{T}$ be the eigenvector corresponding to the eigenvalue $\lambda_{1}^{* *}=0$. Thus $J_{1}^{* *} U^{[11]}=\mathbf{0}$ gives $U^{[11]}=$ $\left(-\left(a_{1}^{*} / a_{11}\right) u_{2}^{[11]}, u_{2}^{[11]}, 0\right)^{T}$, where $u_{2}^{[11]}$ represents any nonzero real numbers. Also, let $W^{[11]}=\left(w_{1}^{[11]}, w_{2}^{[11]}, w_{3}^{[11]}\right)^{T}$ represent the eigenvector corresponding to eigenvalue $\lambda_{1}^{* *}=0$ of $J_{1}^{* * T}$. Hence $J_{1}^{* * T} W^{[11]}=\mathbf{0}$ gives that $W^{[11]}=\left(-\left(a_{21} /\right.\right.$ $\left.\left.a_{11}\right) w_{2}^{[11]}, w_{2}^{[11]},\left(\Psi / a_{11} a_{33}\right) w_{2}^{[11]}\right)^{T}$, where $\Psi=a_{13} a_{21}-a_{11} a_{23}$ is negative due to the sign of the Jacobian elements and $w_{2}^{[11]}$ denotes any nonzero real numbers. Now, since

$$
\frac{d f}{d a_{1}}=f_{a_{1}}\left(Y, a_{1}\right)=\left(\frac{d f_{1}}{d a_{1}}, \frac{d f_{2}}{d a_{1}}, \frac{d f_{3}}{d a_{1}}\right)^{T}=\left(y_{2}, 0,0\right)^{T}
$$

thus $f_{a_{1}}\left(E_{1}, a_{1}^{*}\right)=\left(\tilde{y}_{2}, 0,0\right)^{T}$; hence $\left(W^{[11]}\right)^{T} f_{a_{1}}\left(E_{1}, a_{1}^{*}\right)=$ $-\left(a_{21} / a_{11}\right) w_{2}^{[11]} \widetilde{y}_{2} \neq 0$. So, according to Sotomayor's theorem for local bifurcation the first condition of saddle node bifurcation is satisfied in system (3) at $a_{1}=a_{1}^{*}$. Moreover, substituting $E_{1}, a_{1}^{*}$, and $U^{[11]}$ in (44) gives

$$
\begin{array}{rl}
D^{2} & f\left(E_{1}, a_{1}^{*}\right)\left(U^{[11]}, U^{[11]}\right) \\
& =\left(u_{2}^{[11]}\right)^{2}\left(-2 \frac{a_{2} a_{1}^{* 2}}{a_{11}^{2}},-2 b_{2}, 0\right)^{T} .
\end{array}
$$

Hence, it is obtained that

$$
\begin{aligned}
& \left(W^{[11]}\right)^{T} D^{2} f\left(E_{1}, a_{1}^{*}\right)\left(U^{[11]}, U^{[11]}\right) \\
& \quad=2\left(\frac{a_{2} a_{1}^{* 2} a_{21}}{a_{11}^{3}}-b_{2}\right)\left(u_{2}^{[11]}\right)^{2} w_{2}^{[11]} \neq 0 .
\end{aligned}
$$

Thus, according to Sotomayor's theorem system (3) has a saddle node bifurcation at $E_{1}$ as the parameter $a_{1}$ passes through the value $a_{1}^{*}$; thus the proof is complete.

Theorem 8. Assume that

$$
\begin{aligned}
\left(2 a_{2} y_{1}^{*}+a_{4} y_{3}^{*}+a_{3}\right)\left(2 b_{2} y_{2}^{*}+b_{3} y_{3}^{*}+1\right) & <a_{1} b_{1} \\
y_{1}^{*} & <\frac{a_{1}}{a_{4}} .
\end{aligned}
$$

Then system (3) undergoes a saddle node bifurcation near the interior equilibrium point $E_{2}$, as the parameter $b_{1}$ passes through the bifurcation value $b_{1}^{*}=\Gamma_{1} / \Gamma_{2}$, where $\Gamma_{1}$ and $\Gamma_{2}$ are given in the proof.

Proof. According to the determinant of the Jacobian matrix $J\left(E_{2}\right)$ given by $D_{3}$ in (25), condition (62) represents a necessary condition to have nonpositive determinant for $J\left(E_{2}\right)$. Now rewrite the form of the determinant as follows:

$$
D_{3}=\Gamma_{1}-b_{1} \Gamma_{2} .
$$

Here $\Gamma_{1}=b_{11} b_{23} b_{32}+b_{22} b_{31} b_{13}-b_{11} b_{22} b_{33}-b_{12} b_{23} b_{31}$ and $\Gamma_{2}=b_{13} b_{32}-b_{33} b_{12}$. Obviously, $\Gamma_{1}$ is positive always, while $\Gamma_{2}$ is positive under the condition (63). Thus it is easy to verify that
$D_{3}=0$ and hence $J\left(E_{2}\right)$ has zero eigenvalue, say $\lambda_{2}^{*}=0$, as $b_{1}$ passes through the value $b_{1}^{*}=\Gamma_{1} / \Gamma_{2}$, which means that $E_{2}$ becomes a nonhyperbolic point. Let now the Jacobian matrix of system (3) at $E_{2}$ with $b_{1}=b_{1}^{*}$ be given by

$$
J\left(E_{2}, b_{1}^{*}\right)=J_{2}^{*}=\left(b_{i j}^{*}\right)_{3 \times 3},
$$

where $b_{i j}^{*}=b_{i j} \forall i, j=1,2,3$, and $b_{21}^{*}=b_{1}^{*}$.

Let $U^{[*]}=\left(u_{1}^{[*]}, u_{2}^{[*]}, u_{3}^{[*]}\right)^{T}$ be the eigenvector corresponding to the eigenvalue $\lambda_{2}^{*}=0$. Thus $J_{2}^{*} U^{[*]}=\mathbf{0}$ gives $U^{[*]}=\left(\Phi_{1} u_{3}^{[*]}, \Phi_{2} u_{3}^{[*]}, u_{3}^{[*]}\right)^{T}$, where $\Phi_{1}=\left(b_{12} b_{23}-\right.$ $\left.b_{22} b_{13}\right) /\left(b_{11} b_{22}-b_{12} b_{1}^{*}\right)$ and $\Phi_{2}=\left(b_{1}^{*} b_{13}-b_{11} b_{23}\right) /\left(b_{11} b_{22}-\right.$ $\left.b_{12} b_{1}^{*}\right)$ are positive due to the Jacobian elements and $u_{3}^{[*]}$ represents any nonzero real number. Also, let $W^{[*]}=$ $\left(w_{1}^{[*]}, w_{2}^{[*]}, w_{3}^{[*]}\right)^{T}$ represent the eigenvector corresponding to eigenvalue $\lambda_{2}^{*}=0$ of $J_{2}^{* T}$. Hence $J_{2}^{* T} W^{[*]}=\mathbf{0}$ gives that $W^{[*]}=\left(\Theta_{1} w_{3}^{[*]}, \Theta_{2} w_{3}^{[*]}, w_{3}^{[*]}\right)^{T}$, where $\Theta_{1}=\left(b_{21} b_{32}-\right.$ $\left.b_{22} b_{31}\right) /\left(b_{11} b_{22}-b_{12} b_{1}^{*}\right)$ and $\Theta_{2}=\left(b_{12} b_{31}-b_{11} b_{32}\right) /\left(b_{11} b_{22}-\right.$ $\left.b_{12} b_{1}^{*}\right)$ are negative due to the Jacobian elements and $w_{3}^{[*]}$ denotes any nonzero real numbers. Now, since

$$
\frac{d f}{d b_{1}}=f_{b_{1}}\left(Y, b_{1}\right)=\left(\frac{d f_{1}}{d b_{1}}, \frac{d f_{2}}{d b_{1}}, \frac{d f_{3}}{d b_{1}}\right)^{T}=\left(0, y_{1}, 0\right)^{T}
$$

thus $f_{b_{1}}\left(E_{2}, b_{1}^{*}\right)=\left(0, y_{1}^{*}, 0\right)^{T}$, which gives $\left(W^{[*]}\right)^{T} f_{b_{1}}\left(E_{2}\right.$, $\left.b_{1}^{*}\right)=\Theta_{2} y_{1}^{*} w_{3}^{[*]} \neq 0$. Consequently the first condition of saddle node bifurcation is satisfied. Moreover, by substituting $E_{2}, b_{1}^{*}$, and $U^{[*]}$ in (44) we get that

$$
\begin{aligned}
D^{2} f\left(E_{2}, b_{1}^{*}\right)\left(U^{[*]}, U^{[*]}\right)=2\left(u_{3}^{[*]}\right)^{2} \\
\quad \cdot\left(-a_{2} \Phi_{1}^{2}-a_{4} \Phi_{1},-b_{2} \Phi_{2}^{2}-b_{3} \Phi_{2}, \Phi_{1}+\Phi_{2}-1\right)^{T} .
\end{aligned}
$$

Hence, it is obtained that

$$
\begin{aligned}
& \left(W^{[*]}\right)^{T} D^{2} f\left(E_{2}, b_{1}^{*}\right)\left(U^{[*]}, U^{[*]}\right)=2\left(u_{3}^{[*]}\right)^{2} w_{3}^{[*]} \\
& \quad \times\left[-\left(a_{2} \Phi_{1}^{2}+a_{4} \Phi_{1}\right) \Theta_{1}-\left(b_{2} \Phi_{2}^{2}+b_{3} \Phi_{2}\right) \Theta_{2}\right. \\
& \left.+\left(\Phi_{1}+\Phi_{2}-1\right)\right] \neq 0 .
\end{aligned}
$$

So, according to Sotomayor's theorem, system (3) has a saddle node bifurcation as $b_{1}$ passes through the value $b_{1}^{*}$ and hence the proof is complete.

\section{Numerical Simulations}

In this section, the global dynamics of system (3) is investigated numerically. The objectives first confirm our obtained analytical results and second specify the control set of parameters that control the dynamics of the system. Consequently, system (3) is solved numerically using the following biologically feasible set of hypothetical parameters with different sets 


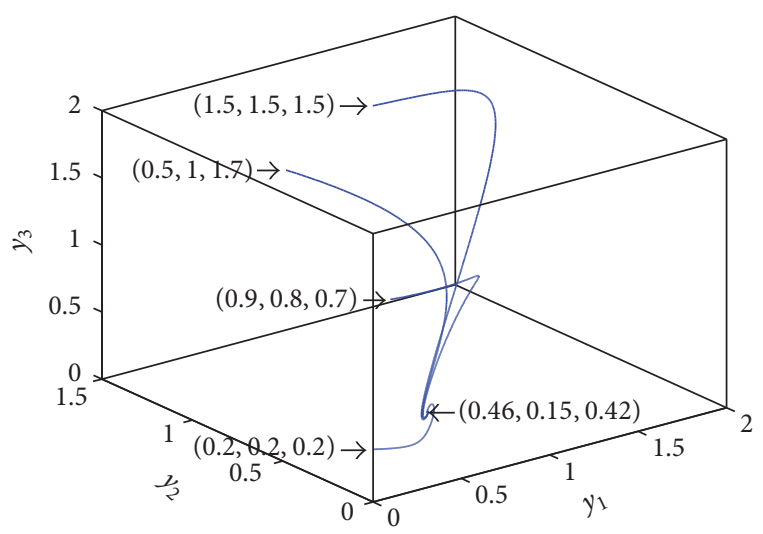

FIgURE 1: 3D phase plot of the system (3) for the data given by (69) starting from different initial values in which the solution approaches asymptotically to $0.46,0.15$, and 0.42 .

of initial points and then the resulting trajectories are drawn in the form of phase portrait and time series figures.

$$
\begin{aligned}
& a_{1}=2, \\
& a_{2}=0.1, \\
& a_{3}=0.4, \\
& a_{4}=0.5, \\
& b_{1}=0.4, \\
& b_{2}=0.1, \\
& b_{3}=0.5, \\
& b_{4}=0.2 .
\end{aligned}
$$

Clearly, Figure 1 shows the asymptotic approach of the solutions, which started from different initial points to a positive equilibrium point $(0.46,0.15,0.42)$, for the data given by (69). This confirms our obtained result regarding the existence of globally asymptotically stable positive point of system (3) provided that certain conditions hold.

Now in order to discuss the effect of the parameters values of system (3) on the dynamical behavior of the system, the system is solved numerically for the data given in (69) with varying one parameter each time. It is observed that varying parameters values $a_{2}, a_{4}, b_{2}, b_{3}$, and $b_{4}$ have no qualitative effect on the dynamical behavior of system (3) and the system still approaches to a positive equilibrium point. On the other hand, when $a_{1}$ decreases in the range $\left(a_{1} \leq 1.05\right)$ keeping other parameters fixed as given in (69) the dynamical behavior of system (3) approaches asymptotically to the vanished equilibrium point as shown in the typical figure given by Figure 2. Similar observations have been obtained on the behavior of system (3) in case of increasing the parameter $a_{3}$ in the range $\left(a_{3} \geq 0.8\right)$ or decreasing the parameter $b_{1}$ in the range $\left(b_{1} \leq 0.2\right)$, with keeping other parameters fixed as given in (69), and then the solution of system (3) is depicted in Figures 3 and 4, respectively. Finally, for the parameters $a_{2}=0.9$ and $b_{4}=0.7$ with other parameters fixed as given in

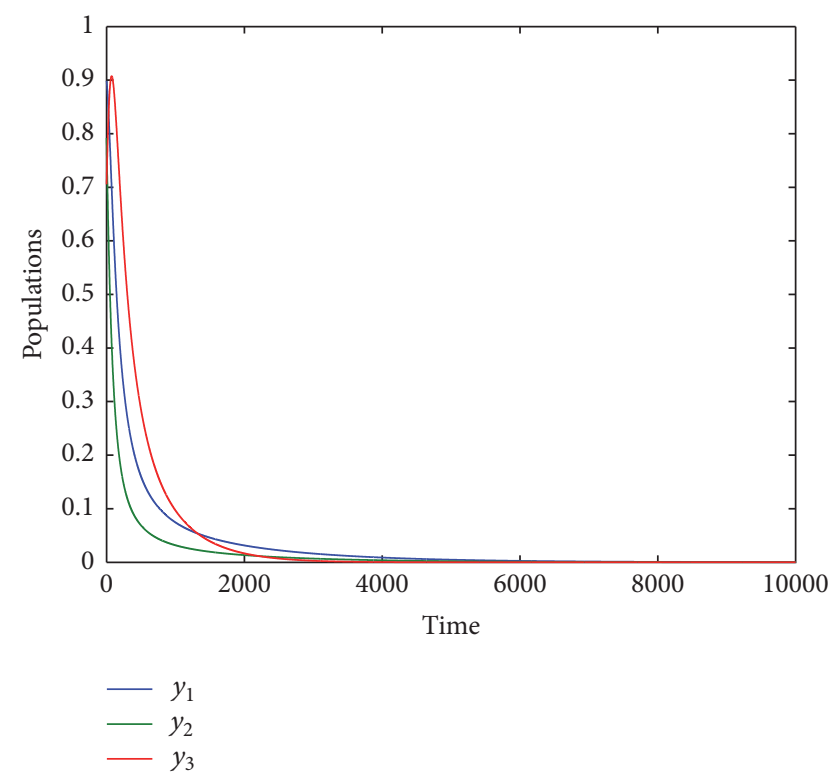

FIgURE 2: Time series of system (3) approaches asymptotically to the vanishing equilibrium point for $a_{1}=0.8$ with other parameters given by (69).

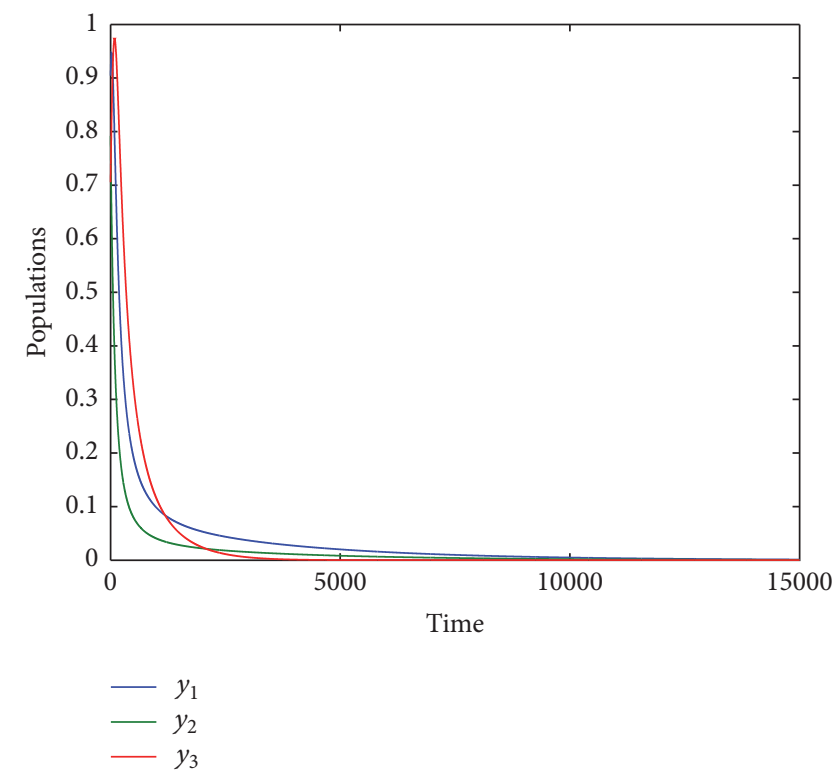

FIGURE 3: Time series of system (3) approaches asymptotically to the vanishing equilibrium point for $a_{3}=0.85$ with other parameters given by (69).

(69), the solution of system (3) approaches asymptotically to the predator free equilibrium point as shown in the Figure 5.

\section{Discussion}

In this paper, a model that describes the prey-predator system having a refuge and stage structure properties in the prey population has been proposed and studied analytically as well as numerically. Sufficient conditions which ensure the 


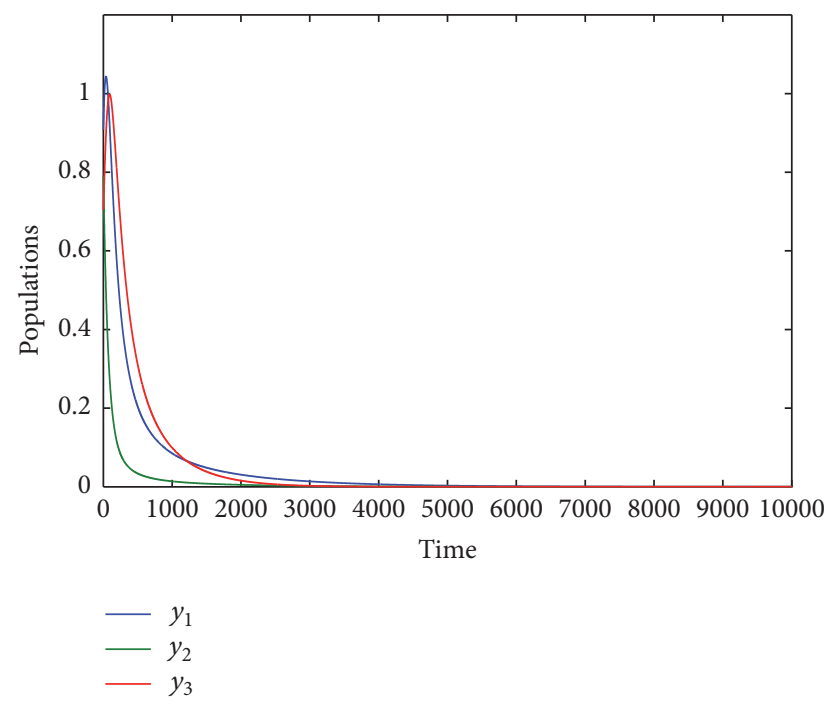

FIGURE 4: Time series of system (3) approaches asymptotically to the vanishing equilibrium point for $b_{1}=0.15$ with other parameters given by (69).

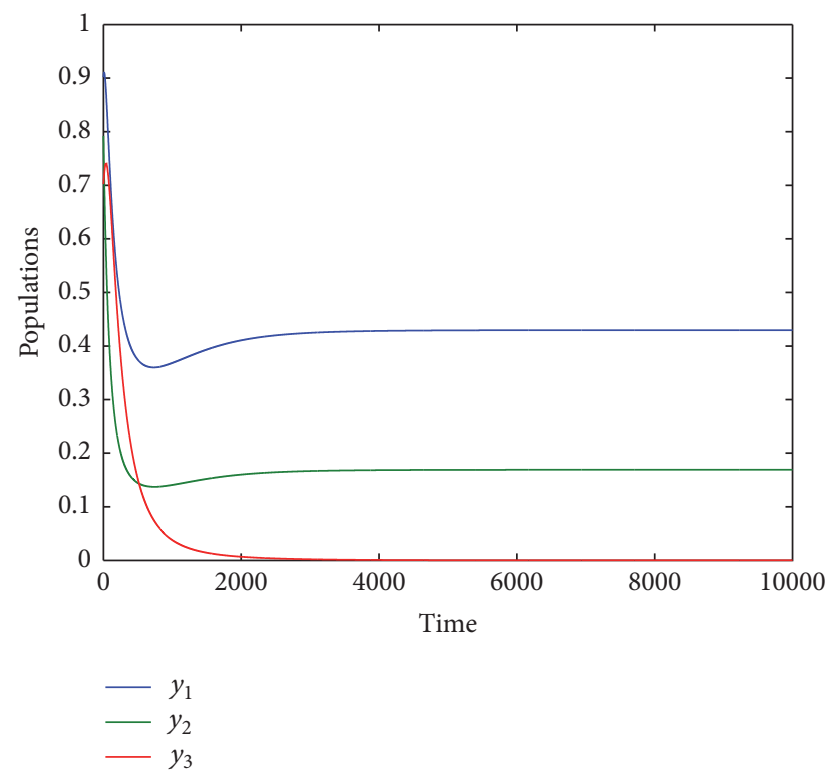

Figure 5: Time series of system (3) approaches asymptotically to the predator free equilibrium point $E_{1}=(0.42,0.16,0)$ for $a_{2}=0.9$ and $b_{4}=0.7$ with other parameters given by (69).

stability of equilibria and the existence of local bifurcation are obtained. The effect of each parameter on the dynamical behavior of system (3) is studied numerically and the trajectories of the system are drowned in the typical figures. According to these figures, which represent the solution of system (3) for the data given by (69), the following conclusions are obtained.

(1) System (3) has no periodic dynamics rather than the fact that the system approaches asymptotically to one of their equilibrium points depending on the set of parameter data and the stability conditions that are satisfied.

(2) Although the position of the positive equilibrium point in the interior of $R_{+}^{3}$ changed as varying in the parameters values $a_{2}, a_{4}, b_{2}, b_{3}$, and $b_{4}$, there is no qualitative change in the dynamical behavior of system (3) and the system still approaches to a positive equilibrium point. Accordingly adding the refuge factor which is included implicitly in these parameters plays a vital role in the stabilizing of the system at the positive equilibrium point.

(3) Decreasing in the value of growth rate of immature prey or in the value of conversion rate from immature prey to mature prey keeping the rest of parameter as in (69) leads to destabilizing of the positive equilibrium point and the system approaches asymptotically to the vanishing equilibrium point, which means losing the persistence of system (3).

(4) Increasing in the value of grownup rate of immature prey keeping the rest of parameter as in (69) leads to destabilizing of the positive equilibrium point and the system approaches asymptotically to the vanishing equilibrium point too, which means losing the persistence of system (3).

(5) Finally, for the data given by (69), increasing intraspecific competition of immature prey and natural death rate of the predator leads to destabilizing of the positive equilibrium point and the solution approaches instead asymptotically to the predator free equilibrium point, which confirm our obtained analytical results represented by conditions (21)-(22).

\section{Competing Interests}

The authors declare that they have no competing interests.

\section{References}

[1] H. I. Freedman, Deterministic Mathematical Models in Population Ecology, vol. 57, Marcel Dekker, New York, NY, USA, 1980.

[2] J. D. Murray, Mathematical Biology, Springer, Berlin, Germany, 3rd edition, 2002.

[3] B. Dubey and R. K. Upadhyay, "Persistence and extinction of one-prey and two-predator system," Nonlinear Analysis: Modelling and Control, vol. 9, no. 4, pp. 307-329, 2004.

[4] S. Gakkhar, B. Singh, and R. K. Naji, "Dynamical behavior of two predators competing over a single prey," BioSystems, vol. 90, no. 3, pp. 808-817, 2007.

[5] T. K. Kar and A. Batabyal, "Persistence and stability of a two prey one predator," International Journal of Engineering, Science and Technology, vol. 2, no. 2, pp. 174-190, 2010.

[6] G. P. Samanta, "Analysis of a delay nonautonomous predatorprey system with disease in the prey," Nonlinear Analysis: Modelling and Control, vol. 15, no. 1, pp. 97-108, 2010.

[7] W. Wang and L. Chen, "A predator-prey system with stagestructure for predator," Computers and Mathematics with Applications, vol. 33, no. 8, pp. 83-91, 1997. 
[8] O. Bernard and S. Souissi, "Qualitative behavior of stagestructured populations: application to structural validation," Journal of Mathematical Biology, vol. 37, no. 4, pp. 291-308, 1998.

[9] X. Zhang, L. Chen, and A. U. Neumann, "The stage-structured predator-prey model and optimal harvesting policy," Mathematical Biosciences, vol. 168, no. 2, pp. 201-210, 2000.

[10] J. Cui, L. Chen, and W. Wang, "The effect of dispersal on population growth with stage-structure," Computers and Mathematics with Applications, vol. 39, no. 1-2, pp. 91-102, 2000.

[11] J. Cui and Y. Takeuchi, "A predator-prey system with a stage structure for the prey," Mathematical and Computer Modelling, vol. 44, no. 11-12, pp. 1126-1132, 2006.

[12] S. Liu and E. Beretta, "A stage-structured predator-prey model of Beddington-DeAngelis type," SIAM Journal on Applied Mathematics, vol. 66, no. 4, pp. 1101-1129, 2006.

[13] S. Xu, "Dynamics of a general prey-predator model with preystage structure and diffusive effects," Computers and Mathematics with Applications, vol. 68, no. 3, pp. 405-423, 2014.

[14] E. González-Olivares and R. Ramos-Jiliberto, "Dynamic consequences of prey refuges in a simple model system: more prey, fewer predators and enhanced stability," Ecological Modelling, vol. 166, no. 1-2, pp. 135-146, 2003.

[15] T. K. Kar, "Stability analysis of a prey-predator model incorporating a prey refuge," Communications in Nonlinear Science and Numerical Simulation, vol. 10, no. 6, pp. 681-691, 2005.

[16] Y. Huang, F. Chen, and L. Zhong, "Stability analysis of a preypredator model with Holling type III response function incorporating a prey refuge," Applied Mathematics and Computation, vol. 182, no. 1, pp. 672-683, 2006.

[17] Z. Ma, W. Li, Y. Zhao, W. Wang, H. Zhang, and Z. Li, "Effects of prey refuges on a predator-prey model with a class of functional responses: the role of refuges," Mathematical Biosciences, vol. 218, no. 2, pp. 73-79, 2009.

[18] V. Krivan, "On the Gause predator-prey model with a refuge: a fresh look at the history," Journal of Theoretical Biology, vol. 274, no. 1, pp. 67-73, 2011.

[19] S. Wang and Z. Ma, "Analysis of an ecoepidemiological model with prey refuges," Journal of Applied Mathematics, vol. 2012, Article ID 371685, 16 pages, 2012.

[20] A. K. Pal and G. P. Samanta, "A ratio-dependent ecoepidemiological model incorporating a prey refuge," Universal Journal of Applied Mathematics, vol. 1, no. 2, pp. 86-100, 2013.

[21] A. De Rossi, I. Ferrua, E. Perracchione, G. Ruatta, and E. Venturino, "Competition models with niche for squirrel population dynamics," in Proceedings of the 11th International Conference of Numerical Analysis and Applied Mathematics( ICNAAM '13), vol. 1558 of AIP Conference Proceedings, pp. 1818-1821, Rhodes, Greece, September 2013.

[22] M. Haque, M. S. Rahman, E. Venturino, and B.-L. Li, "Effect of a functional response-dependent prey refuge in a predator-prey model," Ecological Complexity, vol. 20, pp. 248-256, 2014.

[23] R. Cavoretto, A. De Rossi, E. Perracchione, and E. Venturino, "Reliable approximation of separatrix manifolds in competition models with safety niches," International Journal of Computer Mathematics, vol. 92, no. 9, pp. 1826-1837, 2015.

[24] L. Perko, Differential Equations and Dynamical Systems, vol. 7, Springer, New York, NY, USA, 3rd edition, 2001. 


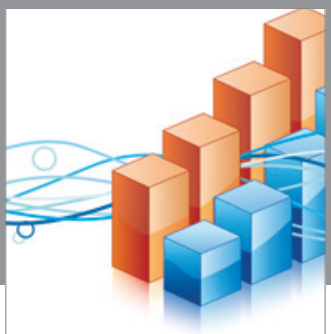

Advances in

Operations Research

vatem alat4

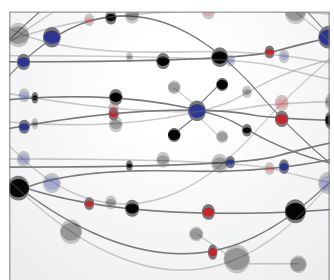

\section{The Scientific} World Journal
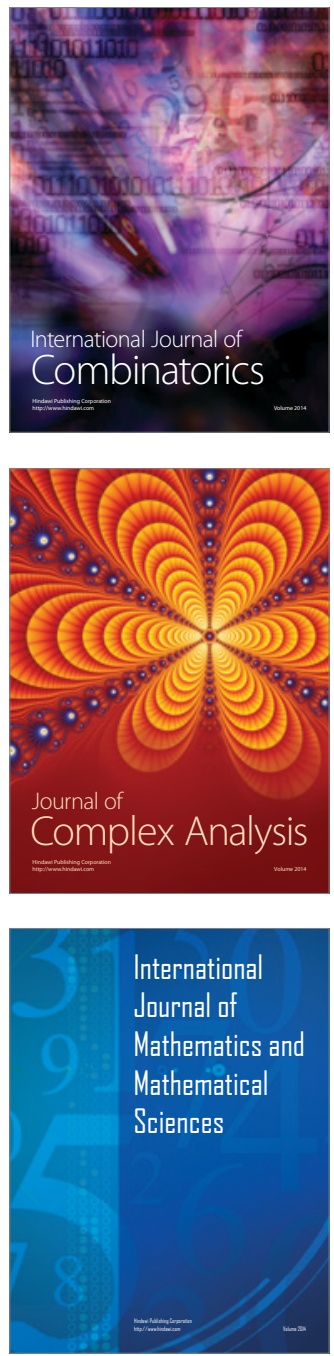
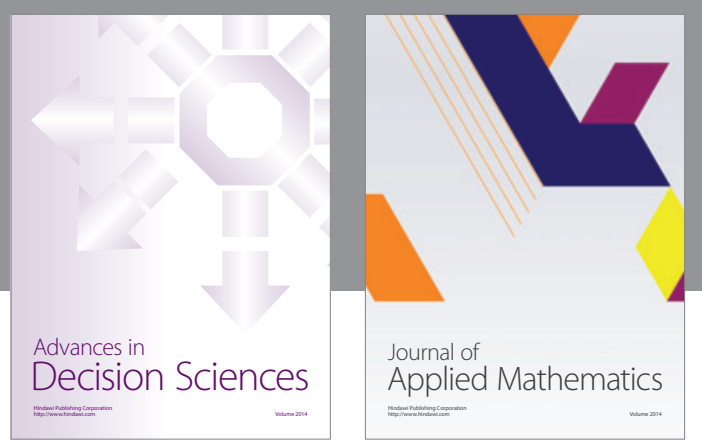

Algebra

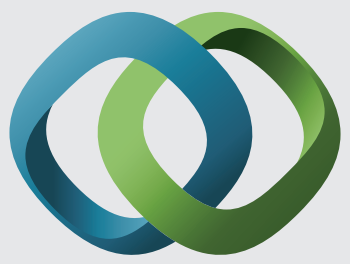

\section{Hindawi}

Submit your manuscripts at

http://www.hindawi.com
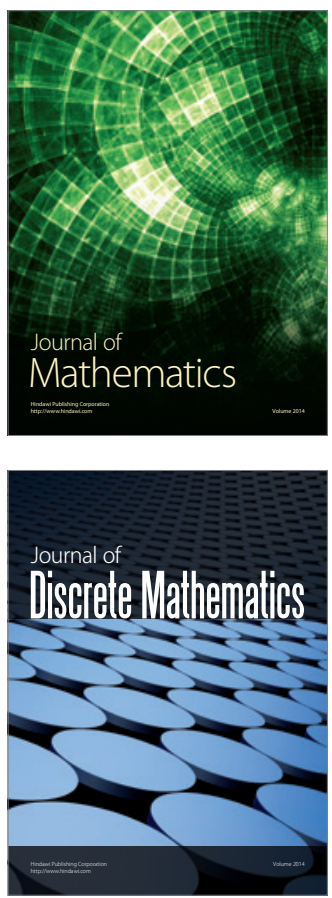

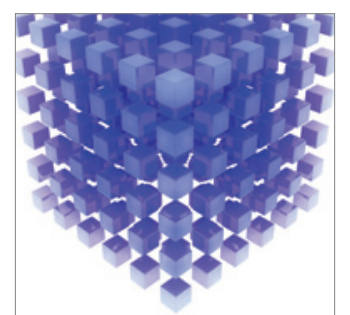

Mathematical Problems in Engineering
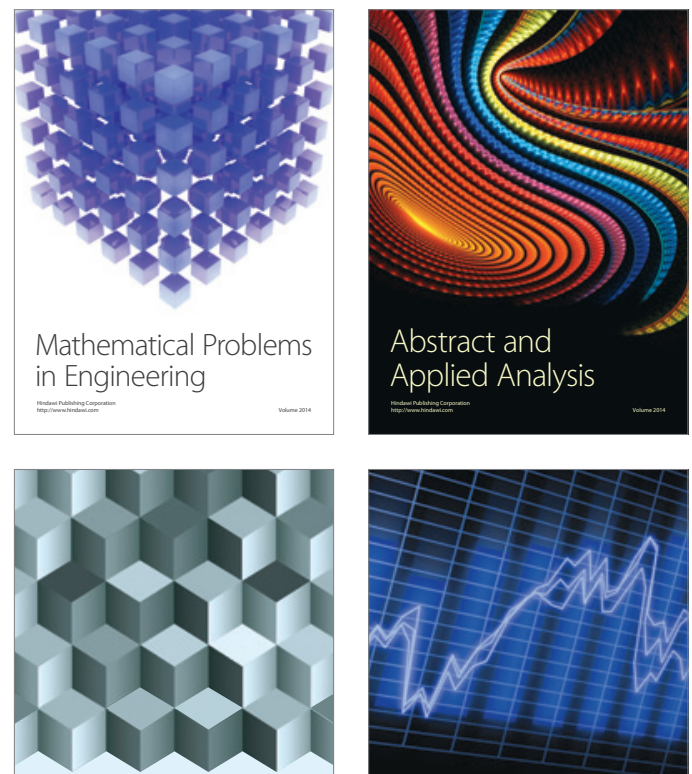

Journal of

Function Spaces

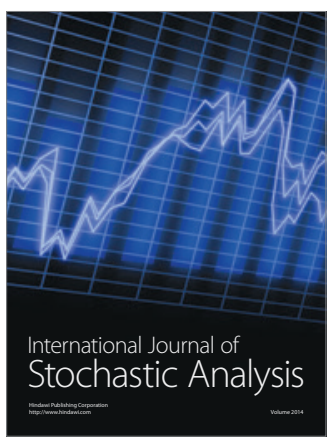

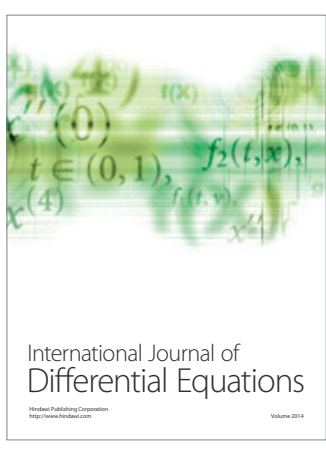
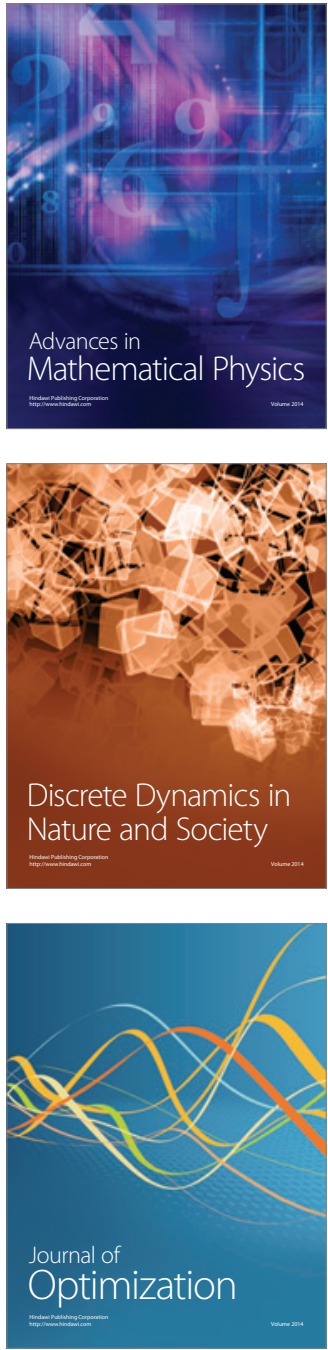